\title{
Design and Synthesis of Immunostimulating Mannosylated Muropeptide Analogs Containing 2-Aminoadamantane-2-carboxylic Acid +
}

\author{
Rosana Ribić ${ }^{1, *}$, Marija Paurević ${ }^{2}$ and Srđanka Tomić ${ }^{3}$ \\ 1 Department of Nursing, University Center Varaždin, University North, Jurja Križanića 31b, HR-42000 \\ Varaždin, Croatia \\ 2 Department of Chemistry, Josip Juraj Strossmayer University of Osijek, Cara Hadrijana 8/A, HR-31000 \\ Osijek, Croatia \\ 3 Faculty of Science, University of Zagreb, Horvatovac 102a, HR-10000 Zagreb, Croatia \\ * Correspondence: rosana.ribic@unin.hr; Tel.: +385-98-183-1206 \\ + Presented at the 2nd Molecules Medicinal Chemistry Symposium (MMCS): Facing Novel Challenges in \\ Drug Discovery, Barcelona, Spain, 15-17 May 2019.
}

Published: 13 August 2019

\begin{abstract}
Muramyl dipeptide (MDP, $N$-acetylmuramyl-L-alanyl-D-isoglutamine) is known as the smallest synthetic adjuvant molecule capable of replacing whole Mycobacteria in Freund's adjuvant. Numerous MDP derivatives were synthesized with the aim to avoid MDP unwanted side-effects. Many of them have therapeutic potential, including clinical use. A very important parameter in the improvement of pharmacological properties of MDP is lipophilicity, e.g., it eliminates drawbacks caused by poor macrophage penetration and rapid elimination. On the other side, mannose receptors (MR), present on immunocompetent cells (such as macrophages and dendritic cells), are considered to be pattern-recognition receptors and responsible for the binding, among others, of mannosylated antigens or relevant biologically active molecules containing mannose, thus affecting the immune reactions. Up to now, our research was directed towards desmuramyl peptides which contain adamantylglycine and mannosylated adamantylglycine moieties bound to the essential part of MDP, L-Ala-D-isoGln. Here, we present the design and synthesis of novel mannosylated muropeptide analogs containing 2-aminoadamantane-2-carboxylic acid. Prepared desmuramyl peptides have lipophilic 2-aminoadamantane-2-carboxylic acid attached at the $\mathrm{N}$-terminus of desmuramy dipeptide core and mannose connected to the tripeptide over a glycolyl linker. Immunostimulating activities of prepared compounds will be evaluated in the mice model using ovalbumin as an antigen and compared with previously prepared derivatives.
\end{abstract}

Keywords: adamantane; adjuvant; mannose; muramyl dipeptide



(C) 2019 by the authors. Licensee MDPI, Basel, Switzerland. This article is an open access article distributed under the terms and conditions of the Creative Commons Attribution (CC BY) license (http://creativecommons.org/licenses/by/4.0/). 